\title{
Editorial
}

\section{Killing cancer cells, twice with one shot}

\author{
ME Bianchi ${ }^{\star, 1}$ \\ Cell Death and Differentiation (2014) 21, 1-2; doi:10.1038/cdd.2013.147
}

I can still remember lecturing that chemotherapy and radiotherapy are effective because they kill cancer cells. That was simple enough, and when I wanted to make it a bit more complex, I ventured into explaining how they mainly killed cancer cells because they divided much more than other cells, and how the killing involved apoptosis. Things have moved on quite a bit since then. Evidence accumulated in the past few years indicates that some anti-cancer drugs do not kill cancer cells, but merely push them into senescence, some do kill cells but not via apoptosis, and some do kill cells via apoptosis but that is not the main ingredient in their efficacy.

The main theme of this issue is that dying cancer cells interact with the immune system, so as to mount a tumorspecific response that contributes, sometimes decisively, to the efficacy of the anti-cancer therapy. Cell death that leads to the release of damage-associated molecular pattern (DAMP) molecules arouses the immune system, and is called immunogenic cell death (ICD, Figure 1). ${ }^{1,2}$ Radiotherapy and specific anti-cancer drugs, notably anthracyclines and oxaliplatin, induce ICD, while other anti-cancer drugs, for example cisplatin, do not. How different modalities of cell death and DAMP release interact with the immune system is reviewed here by Inoue and Tani $^{3}$ and Garg et al., ${ }^{4}$ while the review by Chan et al..$^{5}$ focuses on how natural killer (NK) cells recognize and eliminate stressed cells, including cancer cells that are stressed because of abnormal activation of p53, oncogenes, high levels of DNA damage, chemotherapy or a combination of these cells, and in doing so elicit an immune response.

Several original research papers then delve into the mechanisms that underlie the three major hallmarks of ICD: the release of ATP into the extracellular space, ${ }^{6}$ the exposure of calreticulin on the cell surface, ${ }^{7}$ and the release of high-mobility group box 1 protein (HMGB1). ${ }^{8}$

Calreticulin, also known as calregulin, CRP55, CaBP3, calsequestrin-like protein and endoplasmic reticulum (ER)resident protein 60 (ERp60), is the most abundant soluble protein in the ER, where it acts as a calcium store, and binds to misfolded proteins, thereby preventing them from proceeding to the Golgi and targeting them for degradation. During ER stress, calreticulin is no longer retained in the ER and follows the anterograde flux of vesicles to the plasma membrane. Indeed, ICD requires ER stress. ${ }^{1,2}$ Calreticulin exposed on the cell surface is a potent 'eat-me' signal to macrophages and DCs, and thus promotes the presentation of tumor-associated antigens to T lymphocytes. The paper by Di Giacomo et al. ${ }^{9}$ shows that transglutaminase (TG) 2, a protein with several complex activities, can prevent calreticulin exposure on the cell surface, and therefore TG2-overexpressing cancer cells can escape activation of the immune system. ${ }^{9}$

Curiously, calreticulin is also surface exposed by spermatozoa, both in mammals and nematodes, to facilitate fertilization. Following the phylogenetic trail to its widest extent, Sukkurwala et al. ${ }^{10}$ show that yeast calreticulin, CNE1 protein, is surface exposed following ER stress, and facilitates yeast mating too. ${ }^{10}$ The binding of yeast pheromone $\alpha$-factor to its $\mathrm{G}$ protein-coupled receptor (GPCR) induces CNE1 surface presentation. In mammals, it is ICD-induced chemokines, and in particular IL-8/CXCL8, that by binding to their GPCR receptors promote calreticulin exposure. Concomitant treatment of mouse tumors with cisplatin, which by itself does not induce ICD, and the mouse IL-8 ortholog, Cxcl2, generates an anti-tumor immune response. These data show that calreticulin exposure is not solely a cell-autonomous event, but can actually spread from cell to cell via chemokine communication.

Secretion of ATP, another hallmark of ICD, can work as a 'find-me' signal for DCs, macrophages and their precursors. Pre-mortem autophagy is required for ICD, and for ATP secretion. ${ }^{6}$ Here Martins et al., ${ }^{11}$ using a combination of pharmacological screens, gene silencing and cell imaging, show that a significant pool of ATP is localized in lysosomes in U2OS cancer cells and, upon autophagy activation, can redistribute to autophagosomes and autolysosomes. Autolysosomes can then fuse with the plasma membrane, in a mechanism that requires lysosomal LAMP1, synaptobrevin 1/VAMP1 and caspase activation, but is independent of autophagy. The secretion of ATP further involves the activation ROCK1-mediated, myosin II-dependent cellular blebbing, and requires the opening of pannexin 1 (PANX1) channels, all of them triggered by caspases. Together, these findings suggest that both autophagy and caspase activation and thus some form of apoptosis - play an essential role in ATP release, even though the exact chain of events is still incompletely elucidated.

Ko et al. ${ }^{12}$ examine how radiotherapy works, or sometimes does not work. They show that autophagy increases the resistance of cancer cells to radiotherapy in immunodeficient mice, but decreases it in immunocompetent mice. This is because radiotherapy induces ICD, and autophagy is required for ATP secretion and ICD. Indeed, the inhibition of

\footnotetext{
${ }^{1}$ San Raffaele University and Research Institute, Milano, Italy

${ }^{*}$ Corresponding author: ME Bianchi, Genetics and Cell Biology, San Raffaele University and Research Institute, via Olgettina 58, Milano 20132, Italy. Tel: +39 022643 4765; Fax: +39 022643 5544; E-mail: bianchi.marco@ @sr.it
} 


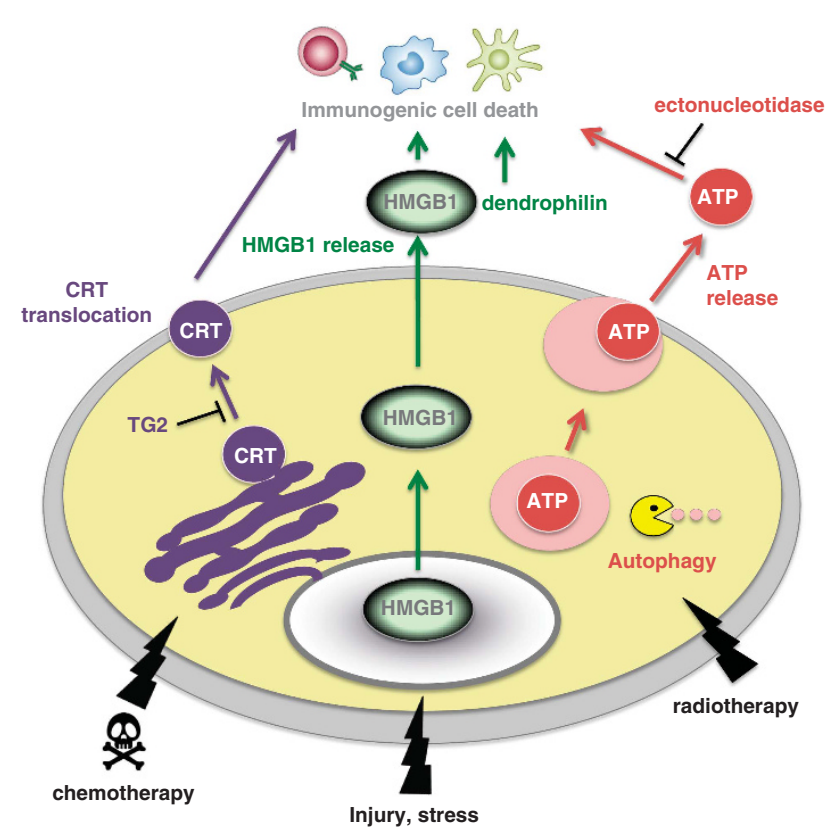

Figure 1 Immunogenic cells death (ICD). Cells can be severely stressed because of an inhospitable environment or condition, or because of tissue injury; they can also be hit by radiotherapy or chemotherapy, which create stress on their own. Cancer cells will eventually die because of treatment, but only if they undergo ICD they will arouse and instruct the adaptive immune system (or, rather, the cellular branch of the adaptive immune system) ${ }^{15}$ against their kin. ICD requires the concomitant release of high-mobility group box 1 protein (HMGB1) and of ATP into the extracellular space, and the exposure of calreticulin (CRT) on the cell surface. Papers in this issue show that ATP is released together with the content of autophago-lysosomes that form during autophagy, and that the inhibition of the degradation of extracellular ATP can partially restore ICD in the absence of autophagy. ${ }^{12}$ Tansglutaminase 2 can interfere with CRT exposure. ${ }^{9}$ Two papers show that CRT exposure can be induced by IL-8 and other cytokines, ${ }^{10}$ and that LPS/dendrophilin can replace extracellular HMGB1 $1{ }^{14}$ as they share the same TLR4 receptor (Artwork by Emilie Venereau).

ecto-ATPases prolongs the activity of what little ATP is secreted when authophagy is disabled, and hence supports ICD induction.

Finally, ICD requires the exodus of HMGB1 from dying cells and its interaction with toll-like receptor (TLR)-4 (ref. 8). HMGB1 is a nuclear protein that is either passively released by dead cells, or actively secreted by stressed or dying cells. However, HMGB1 is retained tightly associated with chromatin when cells die by apoptosis. ${ }^{13}$ Yamazaki et al. ${ }^{14}$ show here that tumors that express a low level of HMGB1 have low immunogenicity even upon ICD, but that immunogenicity can be regained if TLR4 is activated by LPS, or even better by dendrophilin, a highly purified and chemically defined form of LPS. Thus, TLR4 activation and not necessarily HMGB1 are required for ICD. In fact, as signaling downstream of TLR4 requires MyD88 and not TRIF, activation of any TLR might suffice for ICD.

Hannani et al. ${ }^{15}$ show that while the activity of CD4 and CD8 T cells is important for ICD, as previously known, the activity of $B$ cells and the humoral arm of the adaptive immune system is not. Little anti-cancer and anti-calreticulin antibodies are produced following ICD, and their relevance for the anti-tumor response appears minimal. Anthracyclines effectively elicit anti-tumor responses in mice lacking B cells, immunoglobulins or Fc receptors, but not in T-cell-deficient mice. Thus, ICD is almost exclusively a cellular immune response.

The reader will appreciate that several major problems remain to be solved. How exactly do autophagy and apoptosis cooperate in the cell-autonomous program that leads to the exposure of the ATP, HMGB1 and calreticulin trio? How can HMGB1 elicit an anti-tumor response in the form of ICD, and at the same time be protumoral, ${ }^{16}$ such as in mesothelioma development? And how does IL-8 induce calreticulin exposure in neighboring cells, not all of them cancer cells, without inducing autoimmunity? Despite these unknowns, it should be possible to enroll the immune system in the fight against cancer by carefully designing chemotherapy, as reviewed by Bracci et al., ${ }^{17}$ or using viruses, as reviewed by Inoue and Tani. $^{3}$ Injection of dendrophilin or IL-8 into the tumor bed, as suggested by research articles in this issue, ${ }^{10,14}$ are also a distinct possibility. Hopefully, this $C D D$ issue will contribute to stimulating the interest of the scientific and clinical community into what were initially considered curious off-target effects of chemotherapeutic drugs, and in fact may well be a critical element in their efficacy.

1. Kroemer $\mathrm{G}$ et al. Annu Rev Immunol 2012; 31: 51-72.

2. Krysko DV et al. Nat Rev Cancer 2012; 12: 860-875.

3. Inoue H, Tani K. Cell Death Differ 2014; 21: 39-49.

4. Garg AD et al. Cell Death Differ 2014; 21: 26-38.

5. Chan CJ, Smyth MJ, Martinet L. Cell Death Differ 2014; 21: 5-14.

6. Michaud M et al. Science 2011; 334: 1573-1577.

7. Obeid M et al. Nature Med 2007; 13: 54-61.

8. Apetoh L et al. Nature Med 2007; 13: 1050-1059.

9. Di Giacomo G et al. Cell Death Differ (in press).

10. Sukkurwala $A Q$ et al. Cell Death Differ 2014; 21: 59-68.

11. Martins I et al. Cell Death Differ 2014; 21: 79-91.

12. Ko A et al. Cell Death Differ 2014; 21: 92-99.

13. Scaffidi P, Misteli T, Bianchi ME. Nature 2002; 418: 191-195.

14. Yamazaki T et al. Cell Death Differ 2014; $21: 69-78$.

15. Hannani D et al. Cell Death Differ 2014; 21: 50-58.

16. Jube $S$ et al. Cancer Res 2012; 72: 3290-3301.

17. Bracci L et al. Cell Death Differ 2014; 21: 15-25. 